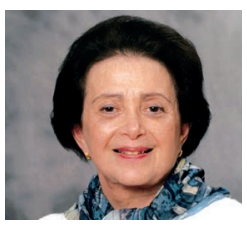

\title{
A heartfelt plea
}

\section{Heart disease is different for women. Researchers must investigate, educate, advocate and legislate to decrease the risks, says Nanette Wenger.}

$\mathrm{U}$ ntil about 30 years ago, heart disease was considered to be a man's problem, even though more women than men died from cardiovascular disease in the United States each year. In the early 1990s, researchers began to investigate the unique aspects of heart disease in women and embrace the concept of sex-specific medicine. Slowly, scientific evidence accumulated that improved the diagnosis and management of cardiovascular disease in women. Translating this evidence into clinical care provided stunning results. In 2000, cardiovascular mortality began to decline in women, and in 2013 , fewer US women than men died of cardiovascular disease - the first time since 1984. Women are delighted to be in second place and hope to remain there.

Not everything is rosy, though. Despite these advances, cardiovascular disease remains the leading cause of mortality for US women and, reversing a favourable earlier trend, there is a concerning increase in the number of deaths among women aged between 35 and 50 . Cardiovascular disease in women continues to be understudied, underdiagnosed and undertreated, and women's awareness of their cardiovascular risk remains suboptimal. To sustain the progress and momentum of the past two decades - and to reverse this rising mortality trend - the research community must do more, including more basic research, population research and clinical research.

Only women face the cardiovascular risk factors associated with pregnancy, including gestational diabetes, gestational hypertension, pre-eclampsia, preterm delivery and giving birth to a child that is small for its gestational age. It is said that the metabolic and physiological changes caused by pregnancy constitute the first major cardiovascular stress test that a woman undergoes. Complications from pregnancy may either unmask an underlying cardiovascular risk or add to it. Either way, pregnancy complications identify women who are at increased risk of future cardiovascular events, and they therefore warrant surveillance and possibly intervention. Yet these women are not being monitored. Obstetric health records are often divorced from other medical records instead of forming part of a woman's risk assessment.

A few cardiovascular diseases affect mainly, or only, women, and these need more research. Spontaneous coronary artery dissection (SCAD) involves a sudden tear in one or more of the arteries that serve the heart, causing a heart attack. It often occurs in premenopausal women, during pregnancy or independently, who have no typical cardiovascular risk factors - and it can kill. But SCAD is poorly understood, and its management is still evolving. Clinicians must become aware of this condition and so they can recognize it when it arises. There is also peripartum cardiomyopathy, which is the development of heart failure at the end of pregnancy or in the weeks after the birth. The heart failure can be treated, but until researchers know what causes it, little can be done to identify women at risk and work towards the ultimate goal of prevention.

In men, the most common form of coronary heart disease is atherosclerotic plaques in the large arteries on the surface of the heart, with the plaque blocking blood flow to the heart (this is known as myocardial ischaemia). But the situation is more diverse in women as well as this 'male' form of coronary disease, they can also have other types that are far rarer in men. Women can have myocardial ischaemia caused by: non-obstructive plaques in these large vessels; damage to the smaller arteries (microvascular disease); spasm in the smaller or larger vessels; or a combination of causes. This complex landscape will require innovative approaches to recognition and treatment.

But women's general and cardiovascular health is not solely a medical issue. When women have 'heart-healthy' lifestyles, their actions influence the behaviours of their family and community, and so benefit the health of nations. Support of women's cardiovascular health must therefore involve more than medical research. The emerging science will be useful only if women have equal access to high-quality, affordable health care, which requires scientists and policymakers to take account of women's beliefs and behaviours; their roles in the community; and their local and global economic, environmental and ethical concerns - as well as of the legislative, political, public policy and sociocultural forces that impinge on their lives. For this to happen, women need equity in research, in prevention, and in access to care and treatments, which can be promoted by raising awareness of how inequity adversely affects women and their families.

Progress came with the US Research for All Act of 2015. The act mandates that the National Institutes of Health ensure that both male and female cells, tissues and animals be included in basic research, the results disaggregated according to sex, and sex differences examined. It also requires that guidelines on including women and minorities in clinical research be updated. Further, the US Food and Drug Administration was directed to ensure that clinical trials of drugs for expedited approval determine their safety and effectiveness for women as well as men - and that the findings of these trials separately examine the outcomes for women and men.

The importance of women enrolling as participants in clinical trials cannot be underestimated. Data for women require women research participants - and the results will provide our daughters and granddaughters with the cardiovascular benefits enjoyed by our sons and grandsons. Excluding elderly patients from clinical trials doubly disadvantages women and must be discontinued, as most coronary events in women occur at an older age. Educating women of all ages about cardiovascular health is paramount. With concerted action, heart disease in women can be reduced dramatically — which will benefit us all.

Nanette K. Wenger is professor of medicine (cardiology) at Emory University School of Medicine, a consultant to the Emory Heart and Vascular Center, and a founding consultant for Emory Women's Heart Center, in Atlanta, Georgia.

email:nwenger@emory.edu 